\title{
EUV lines observed with EIS/Hinode in a solar prominence ${ }^{\star}$
}

\author{
N. Labrosse ${ }^{1}$, B. Schmieder ${ }^{2}$, P. Heinzel ${ }^{3,2}$, and T. Watanabe ${ }^{4}$ \\ ${ }^{1}$ SUPA, School of Physics and Astronomy, University of Glasgow, Glasgow G12 8QQ, Scotland \\ e-mail: Nicolas.Labrosse@glasgow.ac.uk \\ 2 LESIA, Observatoire de Paris-Meudon, 92195 Meudon Cedex, France \\ 3 Astronomical Institute, Academy of Sciences, 25165 Ondřejov, Czech Republic \\ ${ }^{4}$ National Astronomical Observatory of Japan, Mitaka, Japan \\ Received 28 May 2010 / Accepted 6 May 2011
}

\begin{abstract}
Context. During a multi-wavelength observation campaign with Hinode and ground-based instruments, a solar prominence was observed for three consecutive days as it crossed the western limb of the Sun in April 2007.

Aims. We report on observations obtained on 26 April 2007 using EIS (Extreme ultraviolet Imaging Spectrometer) on Hinode. They are analysed to provide a qualitative diagnostic of the plasma in different parts of the prominence.

Methods. After correcting for instrumental effects, the rasters at different wavelengths are presented. Several regions within the same prominence are identified for further analysis. Selected profiles for lines with formation temperatures between $\log (T)=4.7$ and $\log (T)=6.3$, as well as their integrated intensities, are given. The profiles of coronal, transition region, and He II lines are discussed. We pay special attention to the He in line, which is blended with coronal lines.

Results. Some quantitative results are obtained by analysing the line profiles. They confirm that depression in EUV lines can be interpreted in terms of two mechanisms: absorption of coronal radiation by the hydrogen and neutral helium resonance continua, and emissivity blocking. We present estimates of the He II line integrated intensity in different parts of the prominence according to different scenarios for the relative contribution of absorption and emissivity blocking to the coronal lines blended with the He II line. We estimate the contribution of the He II $256.32 \AA$ line to the He II raster image to vary between $\sim 44 \%$ and $70 \%$ of the raster's total intensity in the prominence according to the different models used to take into account the blending coronal lines. The inferred integrated intensities of the He II $256 \AA$ line are consistent with the theoretical intensities obtained with previous 1D non-LTE radiative transfer calculations, yielding a preliminary estimate of the central temperature of $8700 \mathrm{~K}$, a central pressure of $0.33 \mathrm{dyn} \mathrm{cm}^{-2}$, and a column mass of $2.5 \times 10^{-4} \mathrm{~g} \mathrm{~cm}^{-2}$. The corresponding theoretical hydrogen column density $\left(10^{20} \mathrm{~cm}^{-2}\right)$ is about two orders of magnitude higher than those inferred from the opacity estimates at $195 \AA$. The non-LTE calculations indicate that the He ir $256.32 \AA$ line is essentially formed in the prominence-to-corona transition region by resonant scattering of the incident radiation.
\end{abstract}

Key words. Sun: filaments, prominences - line: profiles - Sun: corona - Sun: UV radiation - radiative transfer

\section{Introduction}

Solar quiescent prominences are cool structures $\left(10^{4} \mathrm{~K}\right)$ embedded in the hot corona $\left(10^{6} \mathrm{~K}\right)$. Their formation and long lifetime, of up to a few rotations, are still not well understood. Prominences can be formed by injection of chromospheric plasma or by the condensation of coronal plasma in coronal structures (Mackay et al. 2010). Understanding the dynamics and the thermodynamic properties of the prominence plasma is important to understanding the different stages of the prominence life. Spectroscopy is a very powerful tool, and combined with high spatial and temporal resolutions we can derive information on both the fine structure and dynamics (see Labrosse et al. 2010, for a review of spectral diagnostics and non-LTE modelling techniques).

In the spectral range of the EIS instrument (Extreme ultraviolet Imaging Spectrometer, described in Culhane et al. 2007), several coronal and transition-region (TR) lines are detected (see Young et al. 2007a). In this paper, we show the first EIS spectra obtained in a prominence in this wavelength range and discuss their signatures. There are basically three categories of lines

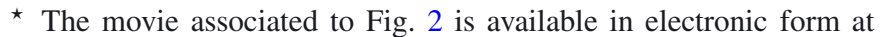
http://www. aanda.org detected by EIS within the prominence: coronal lines, transitionregion lines, and the cool He II $256 \AA$ resonance line. Previous analysis of these lines in the presence of prominences was performed by using mostly filtergrams or integrated intensity rasters (e.g. using the Coronal Diagnostics Spectrometer (CDS), Harrison et al. 1995). Prominence material in the corona may absorb and block the coronal emission and emit transition-region radiation.

Heinzel et al. (2008) studied the two different mechanisms that have to be taken into account to explain the observation of prominences in various EUV coronal lines as dark features in the corona. The lowering of the brightness of EUV coronal lines at the prominence position can be due to two basic mechanisms: absorption and emissivity blocking (formerly called "volume blocking"). The absorption of coronal line radiation at $\lambda<$ $912 \AA$ is due to the photoionisation of hydrogen and both neutral and ionized helium. The lowering of coronal emission observed by Skylab was previously interpreted as being caused by absorption (Orrall \& Schmahl 1976). Anzer \& Heinzel (2005) derived theoretical expressions for the plasma opacity at different wavelengths leading to the determination of the column density of $\mathrm{H} \mathrm{I}$, He I, and He II. The second mechanism is the emissivity blocking discussed by Heinzel et al. (2001) and theoretically developed 
by Anzer \& Heinzel (2005). This blocking, which leads to a lower coronal emission, is due to the presence of a cool prominence plasma (Schwartz et al. 2004; Schmieder et al. 2004) or the low density of the hot plasma because of the existence of a cavity (Wiik et al. 1994; Gibson et al. 2010). Simultaneous multiwavelength images of a prominence at the limb show that the prominence can appear in either emission or absorption depending on the wavelength of the observations. An analysis of different EUV lines can help us to differentiate between these mechanisms and, by inversion, to derive physical quantities such as the opacity and column densities of $\mathrm{HI}_{\mathrm{I}} \mathrm{He}$, and $\mathrm{He}$ II that constrain theoretical models. In Heinzel et al. (2008), this analysis was done using multi-wavelength observations during a coordinated campaign in April 2007 (JOP 178 and HOP 4 ${ }^{1}$ ).

In this paper, we use spectra acquired using the Hinode/EIS spectrometer during the same campaign to show the capability of EIS to help us determine the physical parameters of prominences. The EUV Imaging Spectrometer onboard the Hinode satellite (Kosugi et al. 2007) provides us with the possibility of observing the Sun in two wavelength bands (170-211 and 246-292 A). Each band has a distinct $1024 \times 2048$ pixel CCD detector. We selected the prominence data obtained on April 26, 2007. The capabilities of the EIS spectrometer allow an unprecedented view of the physical conditions within and around the prominence, thanks to the large number of lines detected across a range of temperatures. In the far EUV, this is achieved for the first time with a spatial resolution of around $1^{\prime \prime}$.

The observations are detailed in Sect. 2. In the following sections, we present the rasters obtained by integrating the line profiles, and the line profiles themselves. We discuss the blends of the different lines and how they can be processed to understand the images. The attenuation of the coronal emission in the presence of the prominence is interpreted by absorption and emissivity blocking mechanisms. In Sect. 6, we propose a method to derive the pure emission of the He II $256 \AA$ line, which is blended by several coronal lines. The He II integrated intensity is estimated under different assumptions (different scenarios for the interplay between the coronal blends and the absorption and blocking of the coronal radiation by the prominence plasma). The values obtained are then compared with theoretical values from 1D non-LTE modelling.

\section{Observations}

A prominence surrounded by a large cavity was observed for three days (24-26 April 2007) during the campaign with Hinode (Fig. 1). This was possible because the corresponding filament was extended across more than 30 degrees. During that time, different parts of the filament (footpoints, spine) crossed the disk and were observed as a prominence. On April 24, 2007 , the prominence was already visible on the western limb in the southern hemisphere between 30 and 35 degrees. The prominence did not erupt even during the magnetic flux emergence at the basis of its cavity (Török et al. 2009). The long filament was stable for two more days. Heinzel et al. (2008) studied part of this long filament as a prominence on April 25, 2007. The dynamics of this prominence over the period of observations was studied by Berger et al. $(2008,2010)$, who investigated the vertical flows observed by SOT, and also by Schmieder et al. (2010), who found evidence for flows inclined by an angle of 30 to 90 degrees in the prominence from $\mathrm{H} \alpha$ observations.

\footnotetext{
${ }^{1}$ See http://www.isas.jaxa.jp/home/solar/hinode_op/ hop . php?hop $=0004$
}
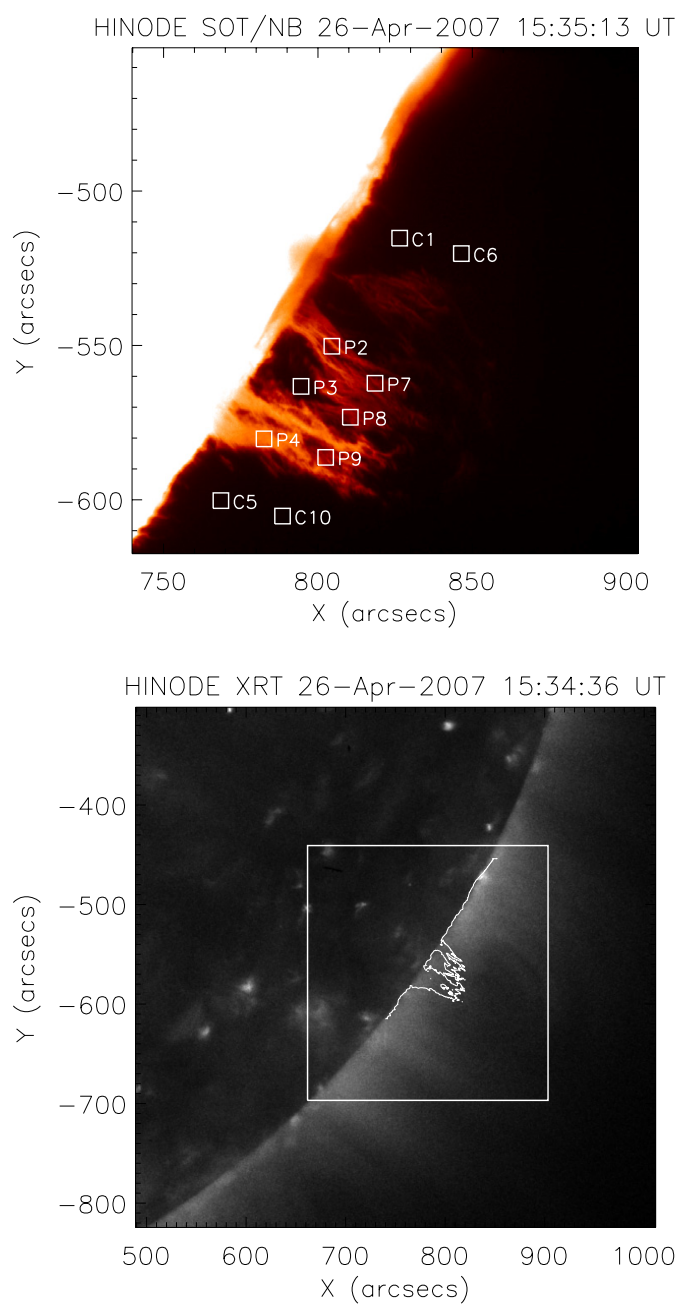

Fig. 1. (Top) $\mathrm{H} \alpha$ observation from Hinode/SOT of the west-limb prominence on 26 April 2007 at 15:35 UT. The boxes on the SOT image indicate the positions around the prominence where the EIS line profiles are studied. (Bottom) Hinode/XRT image showing the prominence cavity. A contour of the $\mathrm{H} \alpha$ limb including the prominence observed with SOT indicates the location of the prominence at the bottom of the cavity. The white box corresponds to the region observed by Hinode/EIS.

We focus here on the last part of the filament. It consists of well-formed off-limb structures seen on April 26, 2007, and is the best observed prominence in the EIS data set for that sequence of observations. Here we analyse data from the fil_rast_s2 study, which uses the 2 " slit and makes a raster of $240^{\prime \prime} \times 256^{\prime \prime}$. At each position of the slit, a spectrum is obtained with a $50 \mathrm{~s}$ exposure. Data from 11 spectral windows is downloaded from the satellite i.e., ten narrow spectral windows, along with a full spectrum on the CCD B of the EIS detector in the long wavelength region, between $189 \AA$ and $211 \AA$ (here referred to as CCDBLONG). This study enables us to observe chromospheric, transition region, and coronal features for a range of temperatures. The selected spectral windows are indicated in Table 1. The formation temperatures of the lines in this table are from Mazzotta et al. (1998) and Young et al. (2007a).

Using the software provided by SolarSoft, we correct the data for several effects (dark current subtraction, cosmic ray removal, flat-field correction, and flagging hot pixels), and convert the number of counts into physical intensity units. Additional corrections to the data consist of internal co-alignment of the two detectors, slit tilt, and orbital variation of the line centroids. 
Table 1. Lines observed in EIS rasters obtained with the fil_rast_s2 study.

\begin{tabular}{|c|c|c|c|}
\hline Ion & Wavelength & $\log (T)$ & Blends \\
\hline Fe VIII & 185.21 & 5.8 & Ni XVII \\
\hline Fe viII & 186.60 & 5.8 & Caxiv \\
\hline CaxvII & 192.82 & 6.7 & $\mathrm{Ov}, \mathrm{Fe} x \mathrm{I}$ \\
\hline Fe XII & 195.12 & 6.1 & Fe XII \\
\hline $\mathrm{Ov}$ & 248.46 & 5.4 & Al VIII \\
\hline $\mathrm{He}_{\text {II }}$ & 256.32 & 4.7 & following four lines \\
\hline Six & 256.37 & 6.1 & \\
\hline $\mathrm{Fex}$ & 256.40 & 6.0 & \\
\hline Fe XII & 256.41 & 6.1 & \\
\hline Fe XIII & 256.42 & 6.2 & \\
\hline Mg vI & 269.00 & 5.7 & \\
\hline Mg vI & 270.40 & 5.7 & $\mathrm{Fe} x \mathrm{xIV}$ \\
\hline Fe xIV & 270.51 & 6.3 & \\
\hline Si viI & 275.35 & 5.8 & \\
\hline $\operatorname{Mg~v}^{a}$ & 276.57 & 5.5 & \\
\hline Mg VII & 278.39 & 5.8 & Si viI \\
\hline Mg VII & 280.75 & 5.8 & \\
\hline
\end{tabular}

Notes. The wavelengths $(\AA)$, temperature of formation (K), and known blends are given. ${ }^{(a)}$ Very weak line.

We use the routines written by P. R. Young available in SolarSoft to correct for these effects.

Figure 1 shows this prominence observed by Hinode/SOT in the $\mathrm{H} \alpha$ line and the surrounding cavity observed by XRT. The temporal evolution of the prominence during the EIS raster between 14:46 UT and 16:29 UT can be seen in the SOT movie associated with on-line Fig. 2. The prominence on that day is more active and changes shape more rapidly than on the previous days. In the movie, we see rather thin and dynamical $\mathrm{H} \alpha$ bright vertical structures separated by large dark lanes reaching a height of $\sim 40000 \mathrm{~km}\left(60^{\prime \prime}\right)$. We selected ten boxes $\left(5 \times 5^{\prime \prime}\right)$ in and around the prominence at two values of the height above the solar limb as seen in $\mathrm{H} \alpha\left(H_{1}=20 \mathrm{Mm}\right.$ and $\left.H_{2}=38 \mathrm{Mm}\right)$, corresponding to different structures of the prominence and its environment. We study the behaviour of the intensities of the different lines observed by EIS in these ten boxes (integrated intensity and profile) in the next sections. The cavity is visible but fainter than on April 25 (Heinzel et al. 2008). It extends to more than $100^{\prime \prime}$ over the limb.

\section{Integrated intensities and rasters}

\subsection{EUV images}

Several interesting lines are present in the EIS observations such as the Fe XII 195.12 $\AA$ line - which is one of the three core lines along with Ca XVII $192.82 \AA$ and He II $256.32 \AA$ that each EIS study must include. Some of the raster images are shown in Fig. 3. The prominence can be most clearly identified with the He II $256.32 \AA$ line, which is the coolest line in this data set. In other cool lines (e.g., O v) with $\log (T)=5.4-5.5$, the prominence is much less visible (not shown here). This is explained by lower count rates on the detector. The signatures of the prominence in the corona are different according to the temperature of formation of the lines. There are basically three categories of lines detected by EIS within the prominence. In coronal lines, the prominence appears to be darker than the surrounding corona, while in TR lines it is brighter than the corona, showing namely the prominence-corona transition region (PCTR). Finally, there is the He II $256 \AA$ resonance line, which is bright

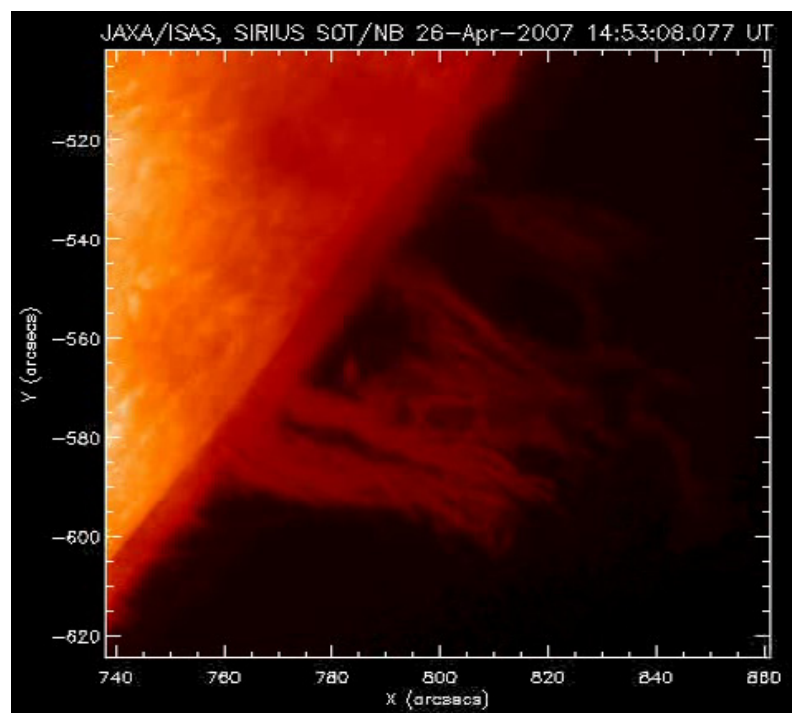

Fig. 2. A still from the Hinode/SOT $\mathrm{H} \alpha$ movie showing the temporal evolution of the prominence observed on 26 April 2007 during the EIS raster between 14:46 UT and 16:29 UT.
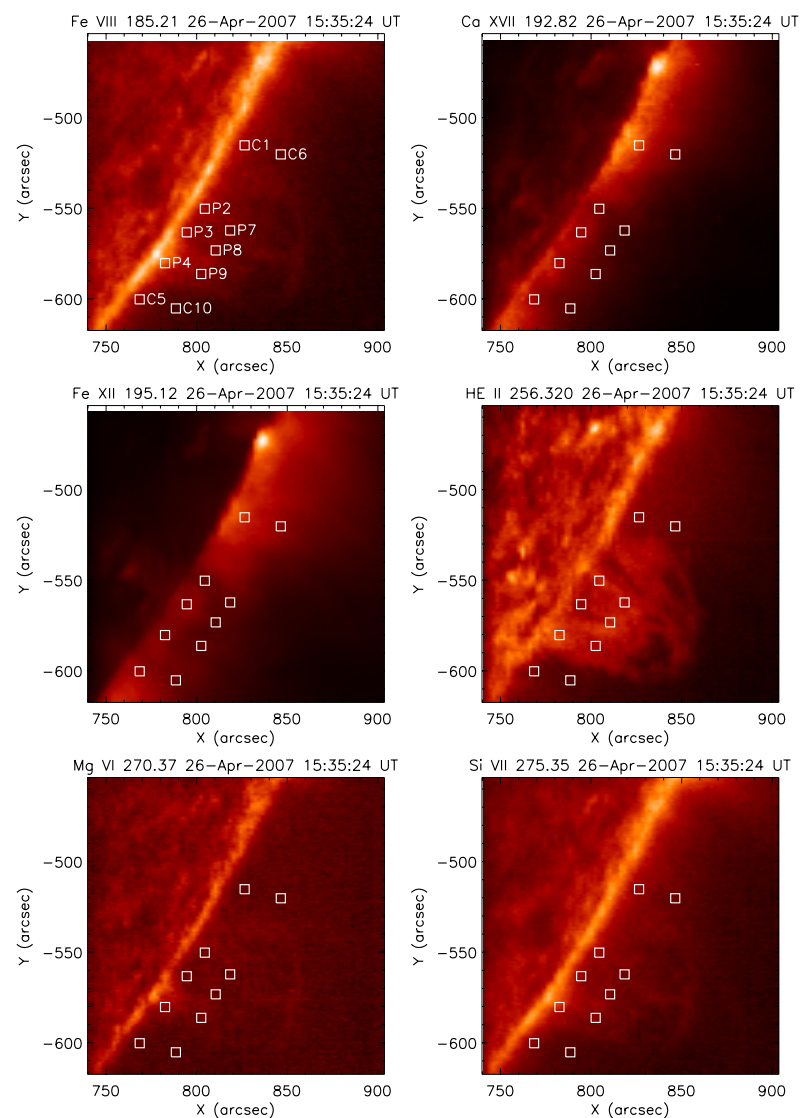

Fig.3. Raster images obtained with fil_rast_s2 on April 26, 2007 between 14:46 UT and 16:29 UT. The white boxes on each raster indicate the positions around the prominence where line profiles are averaged.

on the disk chromosphere and transition region, and is also well detected within the prominence.

On each raster, we show several boxes corresponding to different regions where the line profiles (presented in the following sections) are averaged. The boxes are identified by the letter $\mathrm{C}$ when the box is located in the corona outside the prominence, 
Table 2. Integrated line intensities in various parts of the prominence and its surroundings.

\begin{tabular}{l|ccccc|ccccc}
\hline \hline Line & \multicolumn{7}{|c|}{$H_{1}=20 \mathrm{Mm}$} & \multicolumn{5}{c}{$H_{2}=38 \mathrm{Mm}$} \\
& $\mathrm{C}-1$ & $\mathrm{P}-2$ & $\mathrm{P}-3$ & $\mathrm{P}-4$ & $\mathrm{C}-5$ & $\mathrm{C}-6$ & $\mathrm{P}-7$ & $\mathrm{P}-8$ & $\mathrm{P}-9$ & $\mathrm{C}-10$ \\
\hline Fe VIII 185.21 & 71 & 76 & 84 & 136 & 58 & 35 & 47 & 47 & 56 & 24 \\
& $(101)$ & $(103)$ & $(109)$ & $(161)$ & $(85)$ & $(60)$ & $(66)$ & $(73)$ & $(78)$ & $(45)$ \\
Fe vIII 186.60 & 56 & 61 & 60 & 103 & 44 & 28 & 34 & 37 & 45 & 19 \\
& $(73)$ & $(76)$ & $(78)$ & $(121)$ & $(59)$ & $(43)$ & $(47)$ & $(51)$ & $(57)$ & $(31)$ \\
Ca XVII 192.82 & 216 & 143 & 179 & 190 & 175 & 119 & 95 & 100 & 114 & 102 \\
& $(228)$ & $(151)$ & $(187)$ & $(201)$ & $(181)$ & $(125)$ & $(99)$ & $(103)$ & $(118)$ & $(105)$ \\
Fe XII 195.12 & 927 & 588 & 723 & 691 & 787 & 681 & 520 & 482 & 534 & 575 \\
& $(959)$ & $(604)$ & $(742)$ & $(716)$ & $(805)$ & $(701)$ & $(532)$ & $(495)$ & $(546)$ & $(582)$ \\
O v 248.46 & 44 & 33 & 34 & 46 & 29 & 28 & 27 & 33 & 30 & 20 \\
& $(103)$ & $(99)$ & $(98)$ & $(128)$ & $(104)$ & $(95)$ & $(94)$ & $(96)$ & $(96)$ & $(102)$ \\
He II 256.32 & 360 & 383 & 355 & 489 & 316 & 224 & 323 & 353 & 328 & 191 \\
& $(404)$ & $(423)$ & $(404)$ & $(538)$ & $(355)$ & $(264)$ & $(360)$ & $(392)$ & $(368)$ & $(231)$ \\
Mg vi 270.37 & 39 & 26 & 32 & 34 & 34 & 44 & 32 & 28 & 27 & 30 \\
& $(55)$ & $(42)$ & $(48)$ & $(52)$ & $(50)$ & $(60)$ & $(47)$ & $(43)$ & $(44)$ & $(46)$ \\
Si VII 275.35 & 43 & 46 & 49 & 79 & 36 & 21 & 26 & 31 & 32 & 12 \\
& $(59)$ & $(62)$ & $(67)$ & $(94)$ & $(51)$ & $(37)$ & $(40)$ & $(44)$ & $(49)$ & $(29)$ \\
Mg vII 278.39 & 37 & 40 & 42 & 76 & 31 & 21 & 22 & 27 & 29 & 10 \\
& $(57)$ & $(60)$ & $(63)$ & $(96)$ & $(51)$ & $(39)$ & $(41)$ & $(46)$ & $(47)$ & $(29)$ \\
\hline XRT & 19 & 14 & 14 & 15 & 16 & 13 & 11 & 10 & 11 & 11 \\
\hline
\end{tabular}

Notes. Intensities are in $\mathrm{erg} \mathrm{s}^{-1} \mathrm{~cm}^{-2} \mathrm{sr}^{-1}$ for EIS, and in arbitrary units for XRT. C- and P- correspond respectively to boxes in the corona and in the prominence. P-3 corresponds to the hole seen in $\mathrm{H} \alpha$. For each EIS line, the first row corresponds to intensity values with a constant background removed from the spectrum. The second row (values between brackets) has no background subtraction. The uncertainty in radiometric calibration of EIS data is of the order of $22 \%$ (Lang et al. 2006).

and by the letter $\mathrm{P}$ when the box is on the prominence. $\mathrm{P}-3$ is located in a "hole" of the prominence (a region of the corona almost empty of prominence material) as seen in $\mathrm{H} \alpha$ (see Fig. 1). This kind of void under the prominence evolves rapidly (see Fig. 2 and associated movie).

The analysis of the rasters combined with the line profiles allows us to obtain reliable qualitative as well as quantitative diagnostics. A careful inspection of these images reveals several interesting features.

The He II prominence plasma is in a loop-like shape and reaches higher heights than the $\mathrm{H} \alpha$ vertical structures. Some material can be seen in $\mathrm{He}$ II but not in $\mathrm{H} \alpha$. Is this due to a difference of temperature or density? Do we observe resonance scattering or PCTR emission? Non-LTE models can help us to address these questions. Owing to the blend of this line with several coronal lines, a careful reconstruction (deblending) is needed before any further analysis will be possible (see Sect. 6).

In this data set, the observed lines are formed within a large temperature range, from cool TR (He II at $\log T=4.7)$ to coronal temperatures (Fe xIV at $\log T=6.3$ ) - see Table 1 . The prominence has a cool core (typically 6000 to $8000 \mathrm{~K}$ ) surrounded by a PCTR. The core of the prominence can be seen in emission in $\mathrm{H} \alpha$, while EUV lines are emitted in the PCTR. Coronal line emission in this wavelength range can be absorbed to cause the photoionisation of $\mathrm{He}_{\mathrm{I}}$ for $\lambda<504 \AA$ (He I continuum head) and to cause the photoionisation of $\mathrm{He}$ I and $\mathrm{He}$ II for $\lambda<228 \AA$ (He II continuum head). Over the range of EIS wavelengths, the photoionization of hydrogen is less important (see Anzer \& Heinzel 2005, for all relevant cross-sections). The lack of coronal emission might also be caused by the presence of the cavity (emissivity blocking).

\subsection{Integrated intensities}

To quantify the weakening of coronal lines and the emission of the PCTR of the prominences, integrated intensities were computed in the ten boxes. Table 2 presents the integrated intensities (in erg s $\mathrm{sm}^{-1} \mathrm{cr}^{-1}$ ) for several lines observed by EIS in various parts of the prominence and its surroundings at two values of the height above the solar limb: $H_{1}=20 \mathrm{Mm}$, and $H_{2}=38 \mathrm{Mm}$. For each EIS line, the first row corresponds to intensity values where a constant background was removed from the spectrum. This background is assumed to be of instrumental origin, as it is roughly constant with position for any particular spectral line. The second row has no background subtraction. The XRT intensities (in arbitrary units) are also indicated in Table 2. The full variation in the XRT intensity at the two heights $H_{1}$ and $H_{2}$ is also shown in Fig. 6.

Table 3 presents the ratios of the intensities at the prominence position to that outside in the corona for several EUV TR and coronal emission lines, as well as for the XRT intensities. This allows us to calculate the optical thickness at $195 \AA$ in the same way as in Heinzel et al. (2008) (see Sect. 4.2). The intensity of the TR lines (comparable to those observed by Landi \& Young 2010 ) is enhanced by a factor of 1-2 because of emission from the prominence-corona transition region. The decrease in the intensity of coronal lines is due to the presence of the prominence and the surrounding cavity, and is therefore more significant at low heights where the prominence is denser. We also note that $\mathrm{P}-4$ is in a bright region above the limb.

\section{Coronal lines}

In each panel of Figs. 4 and 5, we plot the profiles of the Ca XVII $192.82 \AA$ and Fe XII $195.12 \AA$ lines for several boxes corresponding to different locations on the prominence $(\mathrm{P}-2, \mathrm{P}-3$, P-4, P-7, P-8, P-9) or in the corona (C-1, C-5, C-6, C-10).

\subsection{Emissivity blocking}

At XRT wavelengths, the absorption by resonance continua is negligible (Anzer et al. 2007) and the low emission in the corona 
N. Labrosse et al.: EUV lines observed with EIS/Hinode in a solar prominence

Table 3. Ratios of the intensities of transition region to coronal lines measured on the prominence and in the corona.

\begin{tabular}{lcccc}
\hline \hline Line & \multicolumn{3}{c}{$H_{1} H_{2}$} \\
& $I(\mathrm{P} 2) / I(\mathrm{C} 1)$ & $I(\mathrm{P} 4) / I(\mathrm{C} 5)$ & $I(\mathrm{P} 7) / I(\mathrm{C} 6)$ & $I(\mathrm{P} 9) / I(\mathrm{C} 10)$ \\
\hline \multirow{3}{*}{ Fe vIII 185.21 } & 1.07 & 2.34 & 1.34 & 2.33 \\
Fe VIII 186.60 & 1.09 & 2.34 & 1.21 & 2.37 \\
Si vII 275.35 & 1.07 & 2.19 & 1.24 & 2.67 \\
\hline \multirow{3}{*}{ Fe XII 195.12 $(r)$} & 0.63 & 0.88 & 0.76 & 0.93 \\
XRT $\left(r_{\mathrm{b}}\right)$ & 0.74 & 0.94 & 0.85 & 1.00 \\
\hline$\tau_{195}(\alpha=0.5)$ & 0.33 & 0.14 & 0.22 & 0.15 \\
$\tau_{195}(\alpha=0.3)$ & 0.62 & 0.24 & 0.39 & 0.27 \\
\hline
\end{tabular}

Notes. $r_{\mathrm{b}}$ is the ratio of coronal emission at a position in the prominence to a position outside in XRT data. The optical thickness at $195 \AA$ A is derived by assuming a spherically symmetric corona with the prominence lying in the plane of the sky $(\alpha=0.5)$ and for an asymmetric corona where the prominence lies behind the plane of the sky $(\alpha=0.3)$.

is due entirely to the presence of the cavity and the prominence itself. The cavity has a low density plasma and the prominence cool plasma blocks a certain volume. A mechanism that can explain the dark void is the emissivity blocking mechanism (Heinzel et al. 2008).

To estimate the level of emissivity blocking, we proceed in the same way as in Heinzel et al. (2008). In the top panel of Fig. 6, we show two cuts in the XRT image, made at constant heights and over about 8 degrees. The relative intensities for these two cuts are displayed in the bottom panel of Fig. 6. We may compare the brightness in the darkest part of the cavity with that at the cavity boundaries (i.e. "quiet" corona). As a result, we get the emissivity-blocking factors $r_{\mathrm{b}}$ shown in Table 3 . The cavity is most pronounced at larger altitudes, as is clearly evident from Fig. 6.

\subsection{Absorption}

The Fe XII line at $195.12 \AA$ has a strong integrated intensity between 495 and $959 \mathrm{erg} \mathrm{s}^{-1} \mathrm{~cm}^{-2} \mathrm{sr}^{-1}$ (background included). This line is broadened because of a blend with another line of Fe XII at $195.18 \AA$, which has the effect of increasing the intensity. The absorption mechanism is efficient at this wavelength and it partially explains the dark features observed in the Fe XII 195.12 raster (Fig. 3). Figure 5 presents the line profiles. The intensity in boxes $\mathrm{C}-1$ and $\mathrm{C}-5$ corresponding to the corona $\left(I_{\mathrm{B}}\right)$, is higher than in the prominence boxes $\mathrm{P}-2-\mathrm{P}-4\left(I_{\mathrm{P}}\right)$. The attenuation of the intensity is due to both the partial absorption of the coronal radiation behind the prominence and the emissivity blocking, which should be taken into account. In the general case, the opacity of the prominence can be estimated by the formula (Heinzel et al. 2008):

$\tau=-\ln \left(\frac{r}{\alpha r_{\mathrm{b}}}-\beta\right)$

where $r=I_{\mathrm{P}} / I_{\mathrm{B}}$ is the ratio of the intensity at the prominence position to that outside at $195 \AA$, and $r_{\mathrm{b}}$ is the ratio of the coronal emission at a position in the prominence to that outside in XRT data (where negligible absorption takes place). The background radiation intensity $I_{\mathrm{B}}$ at the prominence position is that derived from XRT data multiplied by a factor $\alpha$, while the foreground intensity will be a fraction $(1-\alpha)$ of the XRT intensity, and $\beta=(1-\alpha) / \alpha$. Assuming that the coronal line emissivity is symmetrically distributed behind and in front of the prominence, then $\alpha=0.5$. We estimate that the bulk of the prominence is situated behind the plane of the sky, as it had been crossing the limb two days before these observations. Therefore we also estimate the optical depth at $195 \AA$ for the case of an asymmetric corona with $\alpha=0.3$. The optical depth at $195 \AA$ inferred in this way and shown in Table 3 is in good agreement with the values obtained by Heinzel et al. (2008). We note that these results do not depend on the position of our coronal box. As long as the same box is chosen for the reference intensities in XRT and at $195 \AA$, the inferred optical depth at $195 \AA$ in the prominence box will be the same.

The procedure adopted here to derive the optical thickness at $195 \AA$ is similar to the one used later in this work in Sect. 6.1, with the difference that here we distinguish between absorption and emissivity blocking.

The intensity of the Fe xII line in box P-3 (H $\alpha$ void) is larger than in either P-2 or P-4, but is lower than in C-1 and C-5. Is it due to absorption by optically thin prominence plasma that cannot be clearly seen in $\mathrm{H} \alpha$ ? The answer is unclear, even with the information from the intensities of transition region lines such as the Si vII 275 line.

\section{Transition region lines}

\subsection{Fe and Si lines}

The prominence is also clearly identified in emission against the background corona in the Fe VIII 185.21 $\AA$ and Si vII $275.35 \AA$ lines (Fig. 3) formed at $\log (T) \sim 5.8$ (Young et al. 2007b). It is also visible in Fe vIII $186.60 \AA$ (not shown). Corresponding line profiles are shown in Figs. 7 and 8. The Si VII line has no known blend. We note that the shape and intensity of the line profile in box $\mathrm{C}-10$ are very different from those in box C-6.

In these lines, we detect the prominence-to-corona transition region. Although the Fe VIII lines are blended by coronal lines (see Table 1), the intensity of these coronal lines is low enough that we see mainly the emission of Fe vIII lines coming from the PCTR.

\section{2. $M g$ VI line}

The Mg vi 270.40 raster image shows dark features at the location of the PCTR emission region. This is due to the blend with the coronal Fe xIV line at $270.52 \AA(\log (T)=6.3)$. This is clearly seen when looking at the averaged line profiles presented in Fig. 9. It is possible to separate these two lines by 

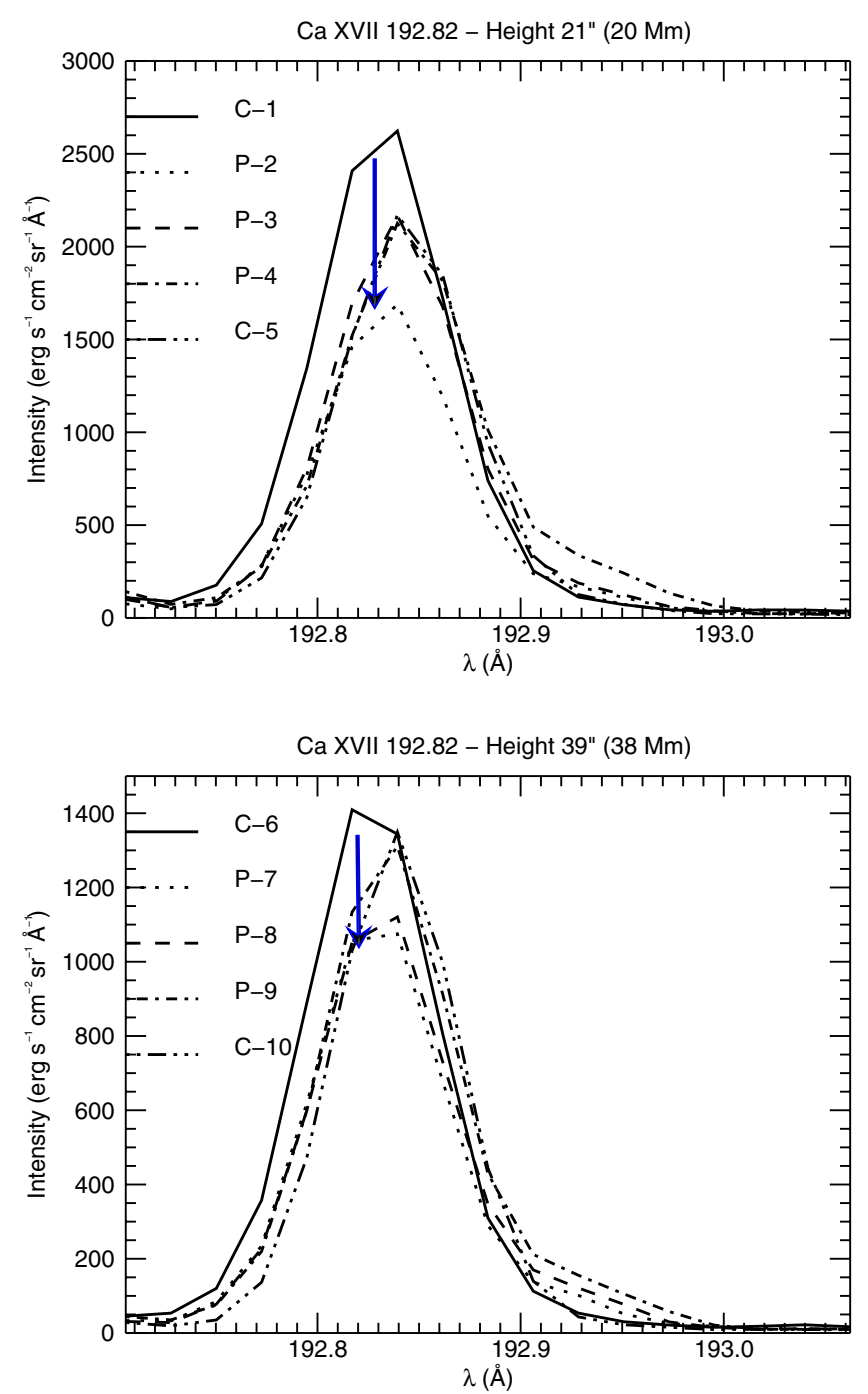

Fig. 4. CaxVII 192 line profiles obtained with fil_rast_s2 on April 26, 2007 between 14:46 UT and 16:29 UT at two heights $(20000 \mathrm{~km}$ and $38000 \mathrm{~km})$. The downward arrows indicate the decrease in line emission from the corona to the prominence. Boxes 1 , 5,6 , and 10 correspond to the corona, while the other ones are located on the prominence.

fitting Gaussian profiles. We find that the prominence is then seen in (weak) emission in the Mg vi line and in absorption in the coronal line of Fe XIV, as expected.

The line profiles in Fig. 9 show that at the lowest altitude in the prominence, the level of emission of the $\mathrm{Mg}$ vI line is not negligible but is still, however, weaker than the contribution of Fe xIv $270.52 \AA$. At greater height, the prominence is fainter, and the emission in the Fe xIV line is strong, but is negligible in the $\mathrm{Mg}$ vi line. Therefore, the image we see in the $\mathrm{Mg}$ vi raster is the result of the integration along the line of sight of the coronal emission in the Fe xIV line, with an additional, small contribution from the Mg vi $270.40 \AA$ line.

\section{He II line and continuum}

The EIS raster in the $256 \AA$ spectral window is shown in Fig. 10 with superimposed contours from the $\mathrm{H} \alpha$ intensity map obtained by SOT. However, the interpretation of the raster images is not straightforward, as a number of blends (of coronal origin)
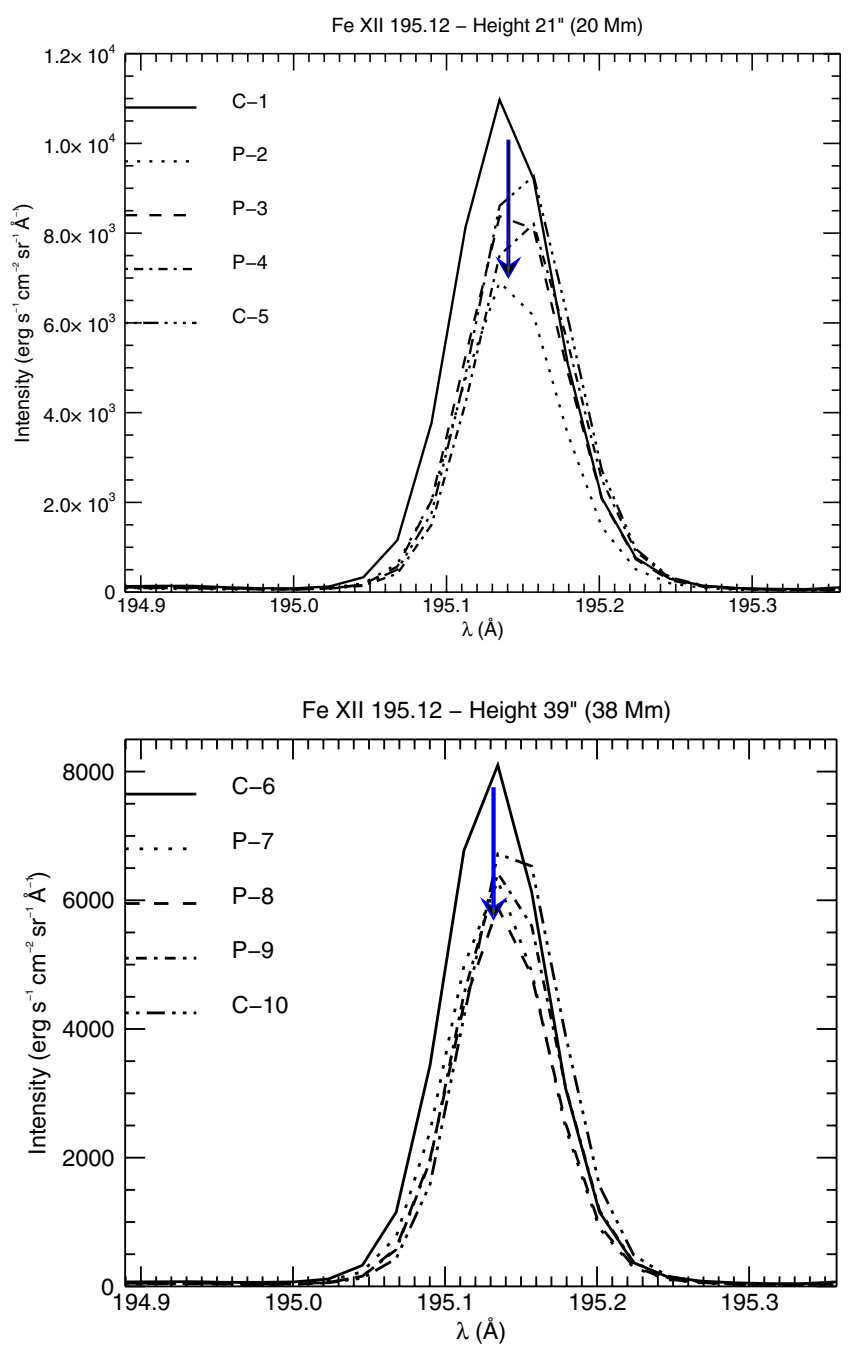

Fig. 5. Same as Fig. 4 for Fe XII 195 line profiles. The downward arrows indicate the decrease in line emission from the corona to the prominence.

with the He II line are present (see Table 1 and the line profiles in Fig. 11).

\subsection{He II 256 Å line deblended}

The He II $256.32 \AA$ line is the Lyman $\beta$ resonance line of ionized helium. It is the only "cool" line observed by EIS. This line, together with the Lyman $\alpha$ line of He II at $304 \AA$ and other He II lines, is important for prominence diagnostics, namely for the determination of the helium ionization degree and the study of the PCTR (e.g. Mariska et al. 1979; Labrosse \& Gouttebroze 2001, 2004). To obtain pure He II 256 line emission originating in the prominence itself, we have to separate it from the He II line emission of coronal origin, from instrumental scattering, and from the coronal line blends. All these components together are referred to here as the 256-blend.

In this paper, we do not intend to reconstruct the true He II line profiles in the prominence, but rather we estimate realistic limits for the integrated line intensities. These are then compared directly with typical model predictions. To avoid problems with coronal brightening close to the limb, and also to compare with the measurements of other authors of the off-limb coronal spectra, we choose the height $H_{2}=38000 \mathrm{~km}$. 
N. Labrosse et al.: EUV lines observed with EIS/Hinode in a solar prominence
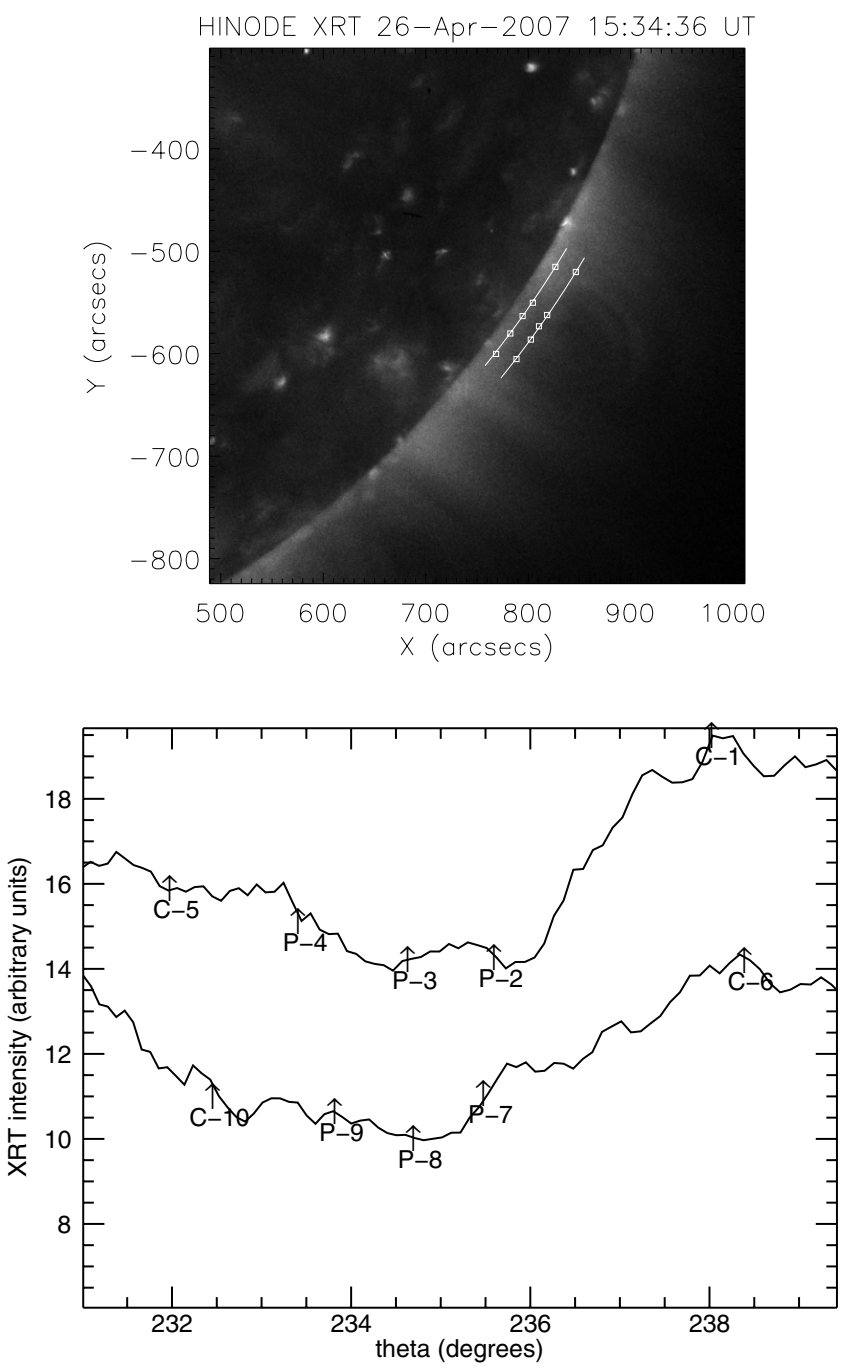

Fig. 6. XRT image (top) and cuts (bottom) through the cavity.

To deblend the coronal 256-blend, we follow the procedure of P. Young (private communication, see also the EISWiki ${ }^{2}$ ) to differentiate between the coronal He II component and coronal blends. He used two Gaussians to fit the whole coronal 256-blend. The first one at 256.321 $\AA$ gives an integrated line intensity $E=37$ (in cgs units), while the other at $256.413 \AA$ gives $E=139$. The total intensity is thus equal to 176 . Therefore, for his EIS coronal observations, the ratio of the two Gaussian components is 0.27 . We did the same for our coronal profiles at boxes C-6 and C-10 and found a ratio around 0.2, similar to Young's value. For coronal blends, Young (EISWiki) gives the ratios of individual blending lines to the total intensity of blends. Similar results were also obtained by E. Landi (private communication), who computed the coronal blends using CHIANTI and the DEM model of the quiet solar corona derived from EIS data by Landi \& Young (2010). The blending lines are listed in Table 1. We are particularly interested in the Six line, which is the strongest blend. Part of its background coronal emission can be absorbed by the prominence due to the expected high He II line opacity (He II Lyman $\beta$ is optically thick as predicted by non-LTE prominence models of Labrosse \& Gouttebroze 2001).

\footnotetext{
${ }^{2}$ http://msslxr.mssl.ucl.ac.uk: 8080/eiswiki/Wiki.jsp? page=HeIIOffLimb, accessed April 2010.
}
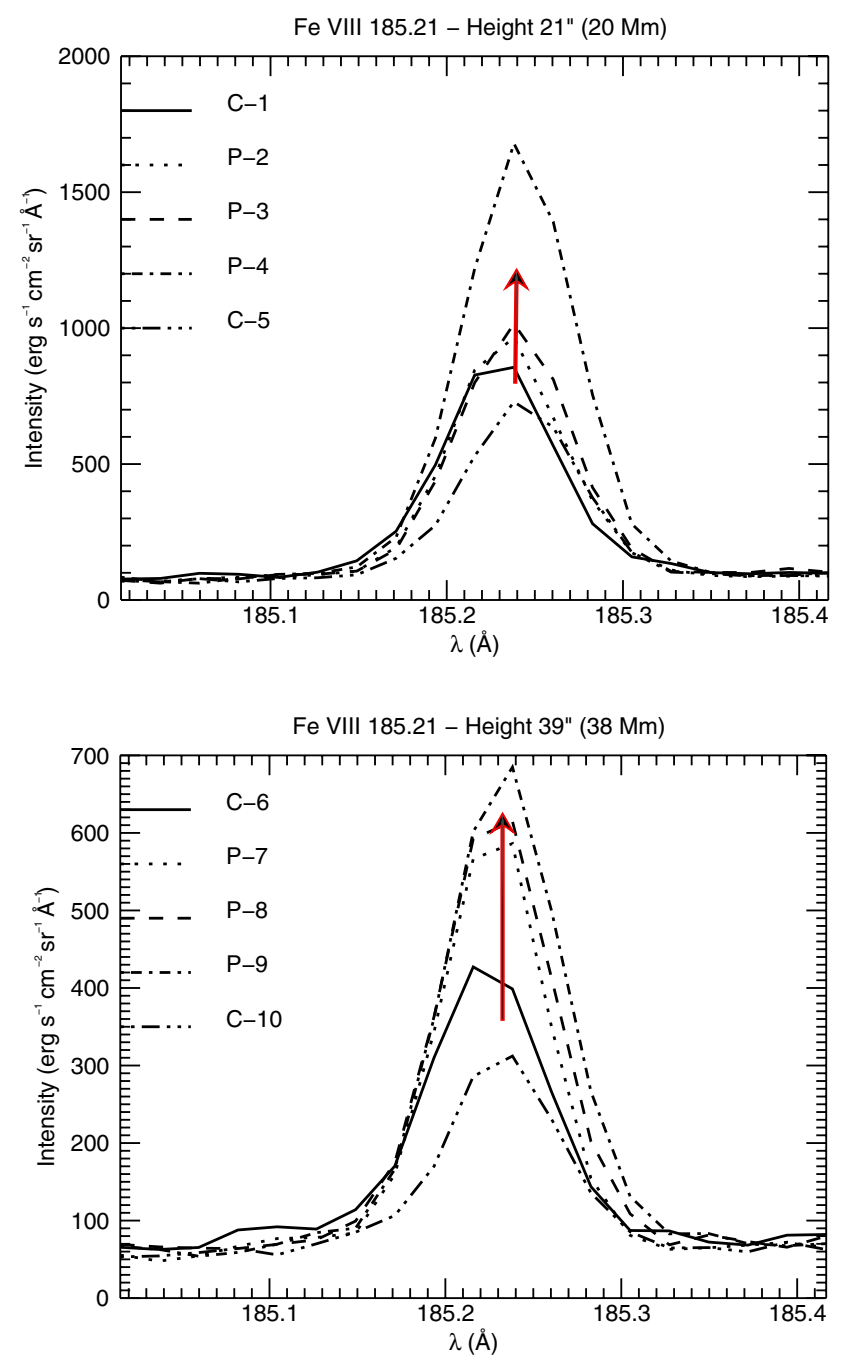

Fig. 7. Same as Fig. 4 for Fe vIII 185 line profiles. The upward arrows indicate the increase in line emission from the corona to the prominence.

All coronal blends are attenuated at the position of the prominence due to (a) absorption by $\mathrm{H}$ I and $\mathrm{He}$ I resonance continua; and (b) emissivity blocking by the prominence and its surrounding cavity (Anzer \& Heinzel 2005; Heinzel et al. 2008). While the emissivity blocking is about the same for similar coronal ions (see Sect. 4.1), the absorption due to photoionization by resonance continua is more complex, depending on the line wavelength and the ionization state of the hydrogen-helium plasma.

The only pure (in the sense that it is not blended by cooler lines) coronal line in our data set is the Fe xII $195.12 \AA$ line. However, the radiation in this line is also absorbed by the He II resonance continuum. To see whether this Fe XII line attenuation, which we directly measure in our EIS data set, can be used to estimate the attenuation of the 256-blend, we computed theoretical attenuation factors at $195 \AA$ and $256 \AA$ using Eq. (11) and Table 2 of Anzer \& Heinzel (2005). We note that the opacities of the $\mathrm{H}_{\mathrm{I}}$ and $\mathrm{He}_{\mathrm{II}}$ resonance continua scale as $\lambda^{3}$, while that of He i scales as $\lambda^{2}$. For a reasonable range of optical thicknesses at these two wavelengths, we derive the corresponding attenuation factors $\frac{1}{2}(1+\exp (-\tau))$. For this, we use the same ionization degrees as in Table 3 of Anzer \& Heinzel (2005). Finally, we arrive at the conclusion that the attenuation factors are quite similar for both wavelengths of interest, the maximum difference being around $10 \%$ for $i=0.5, j_{1}=0.3$, and $j_{2}=0$ (ionization degree 

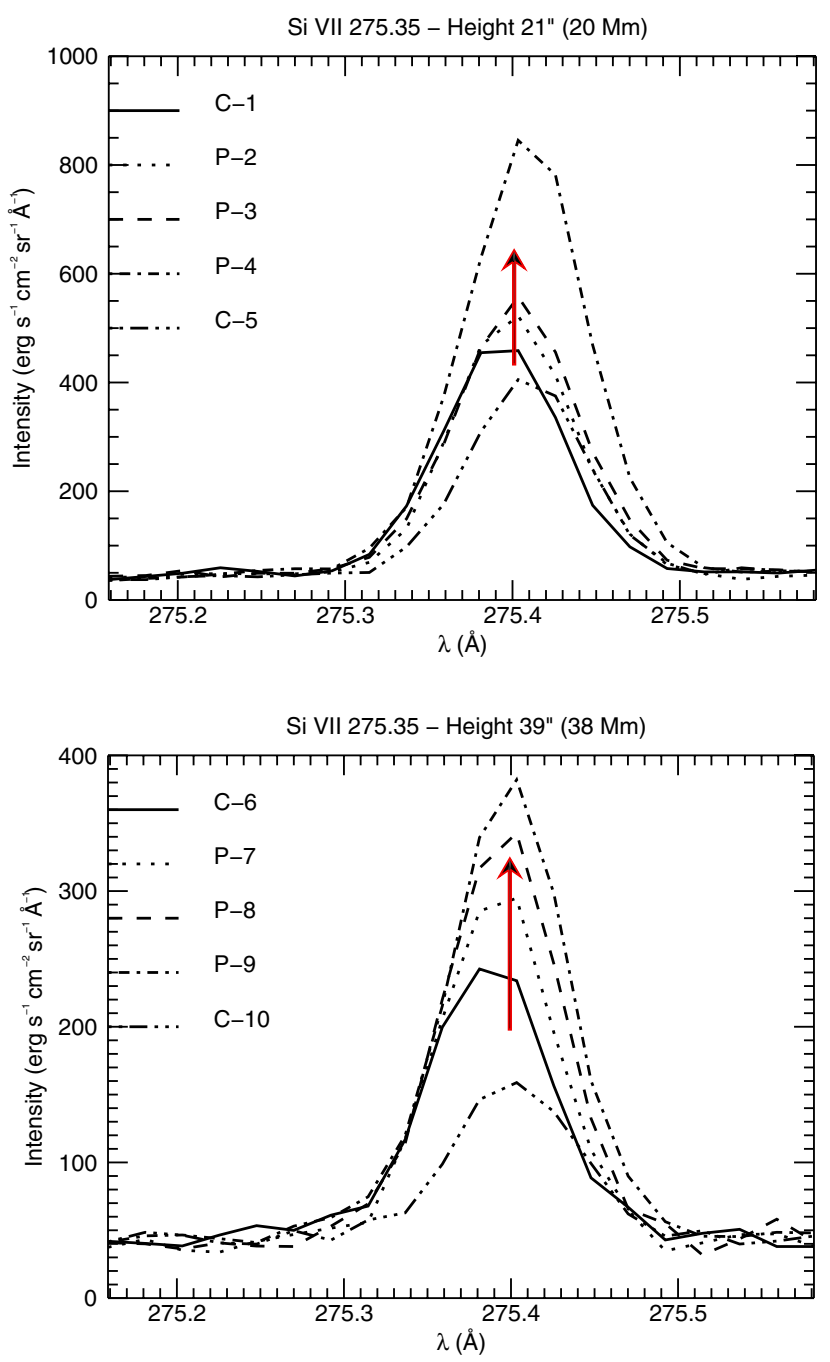

Fig. 8. Same as Fig. 4 for Si vII 275 line profiles. The upward arrows indicate the increase in line emission from the corona to the prominence.

of hydrogen, neutral helium, and ionized helium, respectively see notation in Anzer \& Heinzel 2005).

Therefore, for $256 \AA$ we use the same total attenuation as we can derive from our $195 \AA$ line observations. The latter is the product of $\frac{1}{2}(1+\exp (-\tau))$ and the coronal emissivityblocking factor $r_{\mathrm{b}}$. By this factor, we multiply the coronal blends at boxes $\mathrm{C}-6$ and $\mathrm{C}-10$, for each prominence position. Although the coronal intensities at C-6 and C-10 differ (the latter being lower because of the presence of the cavity), our consistent treatment of the emissivity blocking gives similar results using both boxes, to within a $5 \%$ difference. We therefore take the average values obtained separately for boxes $\mathrm{C}-6$ and $\mathrm{C}-10$. From the total intensity (attenuated) of all coronal blends, we extract the Si $x$ intensity using the ratios given by Young (EISWiki).

To estimate the pure $\mathrm{He}$ II integrated line emission in prominence positions P-7, P-8, and P-9, we use the following four approximate approaches:

Model 1: from the total integrated intensity at the prominence position, we first subtract the full coronal He II, assuming that it is due to an instrumental scattering of the strong disk radiation. We then also subtract all coronal blends, taking properly into account their attenuation.
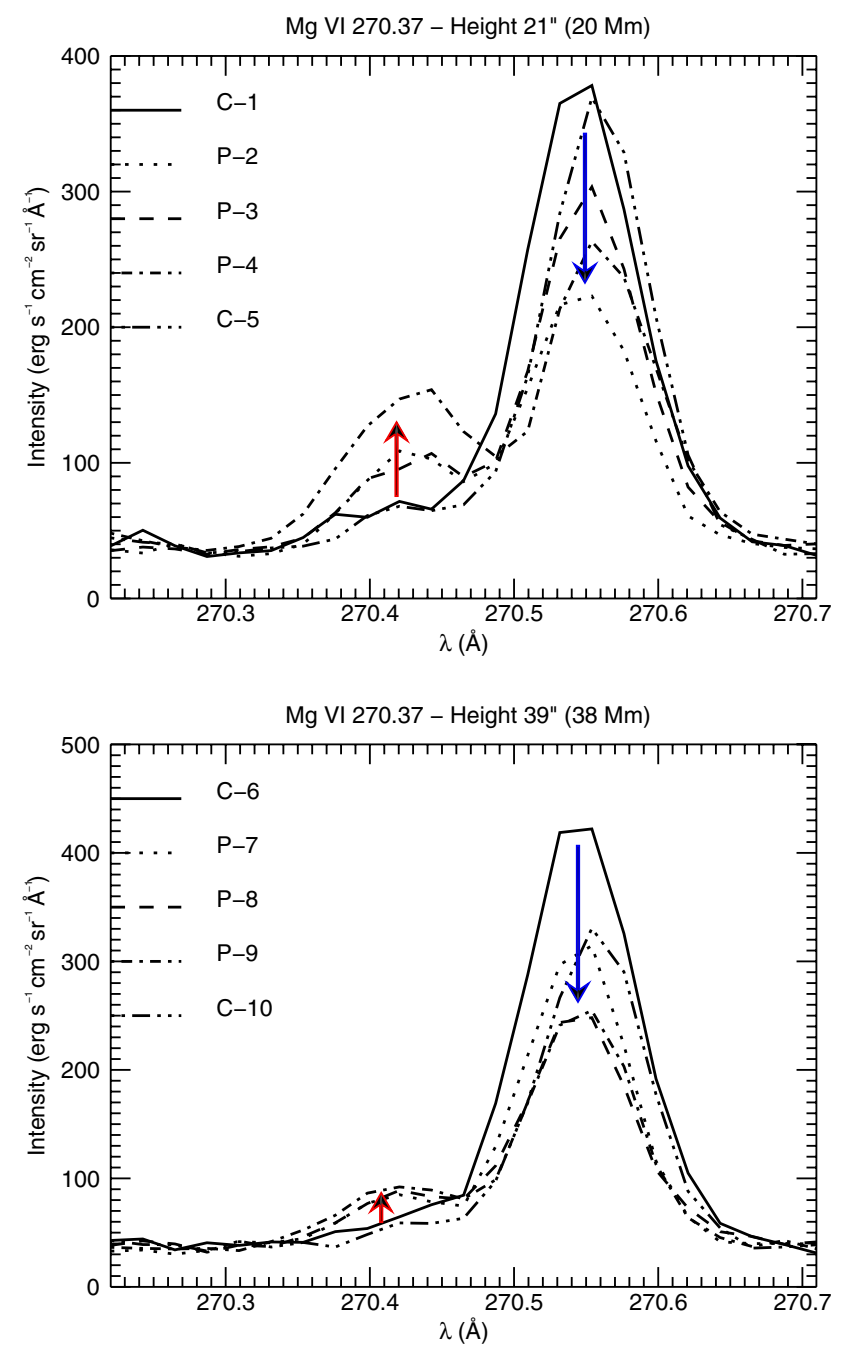

Fig. 9. Same as Fig. 4 for Mg vi and Fe xiv line profiles (blend). Upward and downward arrows indicate an increase in line emission for the $\mathrm{Mg}$ vi line, and the effect of absorption and emissivity blocking due to the prominence in the Fe xIv line.

Model 2: same as model 1, but we add back one half of the Si $x$ blend, assuming that roughly one half (the radiation from behind the prominence) was fully absorbed by the prominence He II line opacity.

Model 3: same as model 1, but we subtract only one half of the coronal He II component. Here we assume that it is of truly coronal origin and thus that one half of it is fully absorbed by the prominence He II line opacity.

Model 4: as model 3, but we add back one half of the Si x blend, assuming that roughly one half was fully absorbed by the prominence He II line opacity.

These four models are aimed at estimating some limiting values of the $\mathrm{He}$ II line intensities in the prominence, thus we ignore some secondary factors. Since we do not reconstruct the true line profiles here, we cannot compute the realistic absorption of the background coronal radiation by the He II line in the prominence, which depends on the relative width and shift of the coronal emission lines and $\mathrm{He}$ II line absorption profile. We thus consider only two limiting cases, i.e. either full absorption or no absorption of the $\mathrm{Si}$ x line radiation. We also do not differentiate 
N. Labrosse et al.: EUV lines observed with EIS/Hinode in a solar prominence

Table 4. Integrated intensities of the He II $256 \AA$ line and the blending lines in different regions of the corona (C) and the prominence (P).

\begin{tabular}{ccccccccc}
\hline \hline Integrated Intensity/Box & P-7 & P-8 & P-9 & C-6 & C-10 & LY(obser) & LY (pred) & Y (obser) \\
\hline Total emission & 323 & 353 & 328 & 224 & 191 & & & 176 \\
He II & & & & 50 & 40 & & 157 & 139 \\
coronal blends & 135 & 125 & 138 & 174 & 151 & & 101 & 95 \\
Si x 256.37 & 92 & 85 & 94 & & & & & \\
1/2 (Si x 256.37) & 46 & 42 & 47 & & & & & \\
Si x 261.04 & & & & & & 104 & 90 & \\
\hline Fe XII 195.12 & 520 & 482 & 534 & 681 & 575 & 581 & 761 & \\
\hline He II (model 1) & 143 & 183 & 145 & & & & & \\
He II (model 2) & 189 & 225 & 192 & & & & & \\
He II (model 3) & 165 & 205 & 167 & & & & & \\
He II (model 4) & 211 & 247 & 214 & & & & & \\
\hline
\end{tabular}

Notes. The total emission quoted here is corrected for the background emission, which is assumed to be of instrumental origin. LY refers to the paper of Landi \& Young (2010) and to CHIANTI computations by E. Landi (private communication), (obser) to observed values, (pred) to predicted values, Y to Young's EISWiki. We note that LY values correspond to a height in the corona of about $25000 \mathrm{~km}$, which is somewhat lower than in the present work.

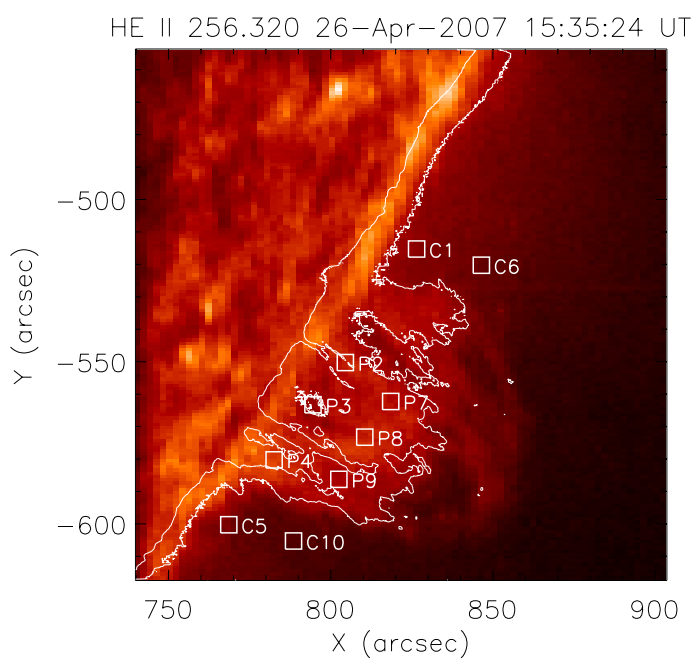

Fig. 10. EIS raster in the $256 \AA$ window with $\mathrm{H} \alpha$ contours from SOT.

between the continuum absorption and emissivity blocking factors (which we did distinguish in Sect. 4.2), and take into account only the combined factor from our $195 \AA$ measurements. To be fully consistent, in model 2 one should subtract the foreground $\mathrm{Si} \mathrm{x}$ radiation that is attenuated only by the emissivity blocking factor, while in model 3 this factor should be used to attenuate one half of the presumably coronal He II radiation (the background part being fully absorbed by the prominence).

Our results for the four models and three prominence boxes are summarized in Table 4. In this table, the blends have been attenuated and the prominence He II line emission estimated using our four models for $H=38000 \mathrm{~km}$. We see that the integrated intensity values are all in a narrow range: 143 to $247 \mathrm{erg} \mathrm{s}^{-1} \mathrm{~cm}^{-2} \mathrm{sr}^{-1}$. As we see in Sect. 6.3, these values can be compared with the non-LTE modelling of Labrosse \& Gouttebroze (2001) and Labrosse \& Gouttebroze (2004). They also represent constraints for new 2D models (Gunár et al. 2008; Gouttebroze \& Labrosse 2009; Léger \& Paletou 2009).

In a future paper, we plan to follow these guidelines and restore the true He II line profiles not only in the prominence, but also in the coronal boxes. One problem is that using two Gaussians for the decomposition of the coronal 256-blend is a rather crude approximation. We performed some preliminary
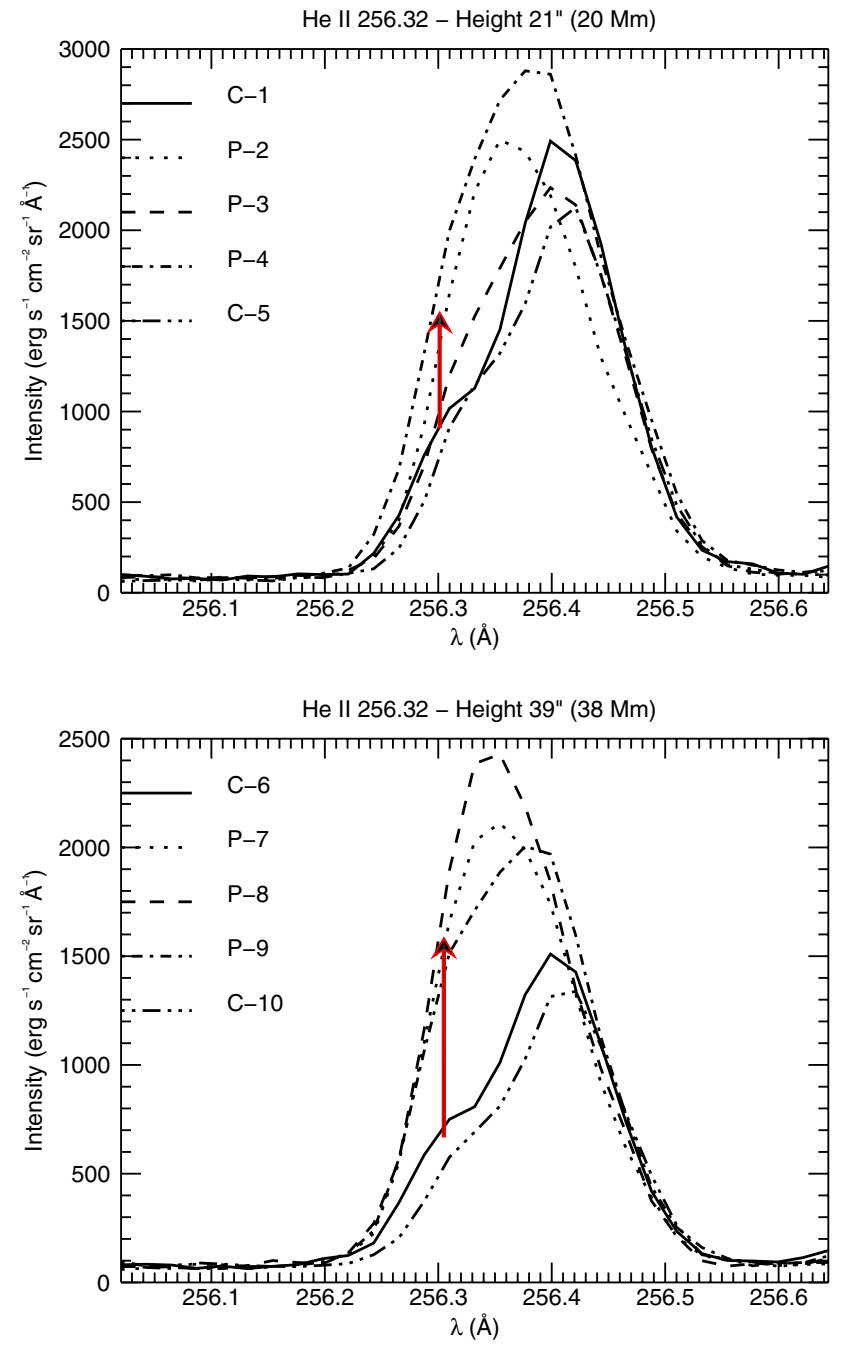

Fig. 11. Same as Fig. 4 for He II 256 line profiles (blend). Upward arrows indicate an increase in line emission in the blue wing due to the prominence.

tests with the detailed profile fitting and obtained a rather asymmetrical and blue-shifted He II coronal component, and a nonGaussian profile of the coronal blend because of a slight rel- 
ative wavelength shift of individual blending lines. Therefore, the detailed profile fitting can lead to a somewhat modified decomposition of the coronal profile. Moreover, we can analyse the He II coronal component profile to investigate its origin more thoroughly. We are able to estimate theoretically the amount of He II coronal radiation caused by the scattering of the disk radiation by coronal He II ions. We are also able to compare the He II coronal component with the shape of the disk emission profile (the raster extends to the disk), and see whether this component resembles the disk one. This would then mean that it could be due to instrumental scattering. Finally, Young (EISWiki) has pointed out that this component of the $256 \AA$ coronal blend could be related to a so far unidentified solar coronal blend or set of blends. In such a case, the de-blending procedure will be the same as when we consider this component to be He II radiation of coronal origin. We postpone this analysis to our next paper.

\subsection{He II continuum}

The continuum window between $189 \AA$ and $210 \AA$ was selected to investigate the level of emission in the helium resonance continua, namely He ir continuum with an edge at $228 \AA$. However, the emission in this continuum below $210 \AA$ is too low in the prominence and no signal can be reliably measured. Using OSO-7 observations, Linsky et al. (1976) measured the intensity at the edge of the He II continuum in a prominence to be a few times $10^{-13} \mathrm{erg} \mathrm{s}^{-1} \mathrm{~cm}^{-2} \mathrm{sr}^{-1} \mathrm{~Hz}^{-1}$. This is fainter than EIS is able to detect in a prominence at $\lambda<210 \AA$. Young et al. (2007b) observed enhanced He II continuum emission in an active region transition region brightening at a level of $\sim 10^{-11} \mathrm{erg} \mathrm{s}^{-1} \mathrm{~cm}^{-2} \mathrm{sr}^{-1} \mathrm{~Hz}^{-1}$.

\subsection{Non-LTE radiative transfer calculations}

As a preliminary analysis, we use a grid of non-LTE models computed by Labrosse \& Gouttebroze (2004) to identify the formation mechanisms of the He II $256 \AA$ line in the prominence (also discussed in Gouttebroze \& Labrosse 2009). In Labrosse \& Gouttebroze (2004), the prominence is represented as a 1D plane-parallel slab standing vertically above the solar surface. Inside the prominence, the pressure and the temperature vary along the line-of-sight according to the theoretical formulae of Anzer \& Heinzel (1999). The pressure profile is derived from the magneto-hydrostatic equilibrium, while the temperature profile is purely empirical. These prominence models include a prominence-to-corona transition region. By using these simple prominence models, we are able to reproduce the range of inferred integrated intensities of the He II $256 \AA$ line reported in Table 4. Here we only compare the theoretical and observed intensities of that line. As an example, Table 5 presents a typical model yielding an integrated intensity in the He II line of $200 \mathrm{erg} \mathrm{s}^{-1} \mathrm{~cm}^{-2} \mathrm{sr}^{-1}$, which is comparable to the inferred value.

From the result of our non-LTE radiative transfer calculations, we estimate that a fraction of about $90 \%$ of the prominence emission in the He II line at $256 \AA$ close to the prominencecorona boundary comes from the scattering of the incident radiation, while the remainder is due to other atomic processes (mainly collisional excitations). This fraction decreases to about $35 \%$ in the central parts of the prominence. Most of the line emission comes from the PCTR (where the He II ions are mostly present) and is due to resonant scattering of the incident radiation. This is consistent with the $\mathrm{H} \alpha$ and $\mathrm{He}$ II 256 lines not
Table 5. Physical parameters for a non-LTE radiative transfer model.

\begin{tabular}{lc}
\hline \hline Parameter & Value \\
\hline Central temperature & $8700 \mathrm{~K}$ \\
Surface temperature & $96500 \mathrm{~K}$ \\
Central pressure & $0.33 \mathrm{dyn} \mathrm{cm}$ \\
Surface pressure & $0.22 \mathrm{dyn} \mathrm{cm}^{-2}$ \\
Column mass & $2.5 \times 10^{-4} \mathrm{~g} \mathrm{~cm}^{-2}$ \\
Hydrogen column density & $10^{20} \mathrm{~cm}^{-2}$ \\
\hline Intensity of the 256 A line & $200 \mathrm{erg} \mathrm{s}^{-1} \mathrm{~cm}^{-2} \mathrm{sr}^{-1}$ \\
\hline
\end{tabular}

always being correlated (as seen in Fig. 1). When the $256 \AA$ line is seen with no $\mathrm{H} \alpha$ counterpart emission, we probably see the PCTR itself (the LOS does not intercept with the central parts of the prominence). In addition, if most of the He II emission in this line results from the scattering of the incident radiation, we do not expect to find a strong correlation with other lines emitted in the PCTR by thermal processes. It is worth noting that our prominence model has a maximum temperature of $10^{5} \mathrm{~K}$ at the coronal boundary, so it cannot be used for an analysis of the other PCTR lines observed by EIS.

Using these results, we estimate the continuum intensity of the hydrogen and helium continua. The predicted He II continuum intensity is lower than $10^{-13} \mathrm{erg} \mathrm{s}^{-1} \mathrm{~cm}^{-2} \mathrm{sr}^{-1} \mathrm{~Hz}^{-1}$ in the spectral range observed by EIS with the CCD B.

Because of the uncertainties attached to the determination of the observed integrated intensity of the He II line, there is some uncertainty regarding the uniqueness of the model presented in Table 5. In particular, the central pressure seems to be high. It is possible that we could obtain a different model with a lower pressure if we took a different temperature profile. On the other hand, we may be seeing the prominence such that the line-of-sight is more or less along the field lines, a situation that our simple 1D slab model cannot reproduce very well. This is related to the value of the hydrogen column density derived from our nonLTE model fitting to the intensity of the He II line alone being larger than expected from the relation between the optical depth at $195 \AA$ and the hydrogen column density given by Eq. (12) in Heinzel et al. (2008). With the values for $\tau_{195}$ reported in Table 3, this relation gives us a hydrogen column density of $n_{\mathrm{H}} \sim 10^{17}-10^{18} \mathrm{~cm}^{-2}$, which is about two orders of magnitude lower than what we obtain from the model of Table 5. Similarly, the hydrogen column density quoted in Table 5 yields $\tau_{195} \sim 16$, a value larger than that derived from the observations (Table 3). On the basis of the modelling developed by Gunár et al. (2008), Berlicki et al. (2011) were able to construct a new model allowing them to reproduce the observed profiles and line intensities of hydrogen Lyman and $\mathrm{H} \alpha$ lines observed in the same prominence with 40 threads of temperature $8000 \mathrm{~K}$, central pressure $0.035 \mathrm{dyn} \mathrm{cm}^{-2}$, and a column mass $3 \times 10^{-6} \mathrm{~g} \mathrm{~cm}^{-2}$ across the field lines. With 40 threads, this yields a total column mass of $1.2 \times 10^{-4} \mathrm{~g} \mathrm{~cm}^{-2}$ at the centre of the prominence, only a factor of two less than our value (Table 5).

That we obtain different results in our modelling is not very surprising. Berlicki et al. used a complete set of hydrogen lines to find a 2D model that reproduces well their observed line profiles. In the present study, our 1D model is simply based on the estimated integrated intensity of the He II line at $256 \AA$. If anything, this highlights the need to consistently use the information derived from both the hydrogen and helium line profiles. We made some preliminary attempts to combine both types of models to compute the intensity of the He II 256 line using the 
Berlicki et al. models, but the resulting integrated intensity is too low. This may be a signature of a new diagnostic of the PCTR provided by the He II lines.

We also stress that this prominence differs from the prominence observed the previous day and analysed in Heinzel et al. (2008). In particular, we do not see the same absorption for the prominence in EIS images because there are coronal structures in the foreground. STEREO A and B effectively detect coronal structures in $171 \AA$ projected inside the cavity. This may in turn affect our estimates of the optical depth at $195 \AA$.

In our next paper, we will attempt to recover the full line profile of the He II $256 \AA$ line in the prominence from the EIS data, and we will make a more detailed quantitative comparison with predicted line profiles. We will also study the other He lines predicted by our models.

\section{Conclusions}

The EUV Imaging Spectrometer of Hinode has been found to be a powerful instrument that is providing new insights into the physics of solar prominences, and in particular of the prominence-to-corona transition region. Despite the EIS sensitivity being tuned towards short wavelengths bands, several lines are suitable for investigating the properties of the prominence region and the surrounding corona in terms of temperature and density.

In particular, the He II $256.32 \AA$ line is a strong emission line that contains information about the prominence fine structure.

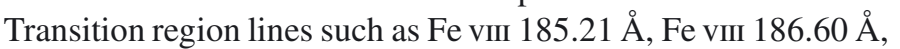
and Si vII $275.35 \AA$ are also well-observed in the prominence. Coronal lines clearly reveal the prominence as a dark feature because of both the absorption of the coronal EUV radiation by the resonance continua of hydrogen and helium, and the emissivity blocking effect. We have used the intensities observed by both EIS at $195 \AA$ and XRT to estimate the optical depth at $195 \AA$ in the prominence.

Although the He II 256.32 A line is listed with a temperature of formation of about $50000 \mathrm{~K}$ in Mazzotta et al. (1998), this value should not be taken as representative of the prominence plasma seen in Fig. 3. The prominence plasma is optically thick at this wavelength, and the resonant scattering of the incident radiation coming from the solar disk and the surrounding corona is therefore important. This situation is similar to that for the first resonance line of ionised helium at $303.78 \AA$ (Labrosse \& Gouttebroze 2001; Labrosse et al. 2008). This provides constraints on new 2D models (see Gunár et al. 2008; Gouttebroze \& Labrosse 2009; Léger \& Paletou 2009).

Off-limb raster images from EIS should be analysed carefully to distinguish the contributions of the different lines blended with the nominal line. The interpretation of the He II raster image is not straightforward. In this paper, we have analysed the blend of the He II $256 \AA$ line and obtained a reasonable estimate of the He II line integrated intensities in several prominence locations. These are consistent with results of non-LTE modelling (Labrosse \& Gouttebroze 2004). However, the corresponding theoretical hydrogen column density is about two orders of magnitude higher than what is inferred from the opacity estimates at $195 \AA$.

The detailed analysis of the line profiles of the 256-blend will be studied in a future paper. We will also address the comparison of the diagnostics obtained from the $256 \AA$ line with those from the $304 \AA$ line of He II. We do not expect to find a strong correlation between the two lines in our theoretical results. Observational studies (e.g., Glackin et al. 1978) have found that both lines were uncorrelated in the quiet Sun. We will also investigate whether the He II $256 \AA$ line could be used in conjunction with other lines to estimate the $\mathrm{He}$ I/ $/ \mathrm{He}$ I abundance.

The robustness of the spectral diagnostics of the filaments and prominences observed for JOP 178 will be enhanced by the large number of lines observed by the three space-based spectrometers EIS, CDS, and SUMER, from the ground-based $\mathrm{H} \alpha$ data, and from our non-LTE radiative transfer codes to analyse the hydrogen (Gouttebroze \& Labrosse 2000; Gunár et al. 2007) and helium (Labrosse \& Gouttebroze 2001, 2004) spectra. We plan to combine these observations to more tightly constrain our non-LTE models and infer the thermodynamical properties of the prominences and filaments and their surroundings.

Acknowledgements. We thank the referee for their constructive comments. We are grateful to Giulio Del Zanna, Stanislav Gunàr, Pierre Gouttebroze, Enrico Landi, and Peter Young for helpful discussions, as well as to our colleagues from the International Teams 123 and 174 of the International Space Science Institute (ISSI) in Bern. The support of ISSI is acknowledged. N.L. was partially supported by the European Commission through the SOLAIRE Network (MTRNCT-2006-035484) and by STFC Rolling Grant (ST/F002637/1). P.H. was partially supported by the ESA-PECS project No. 98030. He also acknowledges the hospitality and support of the Paris Observatory. We thank Pavol Schwartz for helping us with Fig. 6. Hinode is a Japanese mission developed and launched by ISAS/JAXA, with NAOJ as domestic partner and NASA and STFC (UK) as international partners. It is operated by these agencies in co-operation with ESA and NSC (Norway). This research has made use of NASA's Astrophysics Data System.

\section{References}

Anzer, U., \& Heinzel, P. 1999, A\&A, 349, 974

Anzer, U., \& Heinzel, P. 2005, ApJ, 622, 714

Anzer, U., Heinzel, P., \& Fárnik, F. 2007, Sol. Phys., 242, 43

Berger, T. E., Shine, R. A., Slater, G. L., et al. 2008, ApJ, 676, L89

Berger, T. E., Slater, G., Hurlburt, N., et al. 2010, ApJ, 716, 1288

Berlicki, A., Gunár, S., Heinzel, P., Schmieder, B., \& Schwartz, P. 2011, A\&A 530, A 143

Culhane, J. L., Harra, L. K., James, A. M., et al. 2007, Sol. Phys., 243, 19

Gibson, S. E., Kucera, T. A., Rastawicki, D., et al. 2010, ApJ, 724, 1133

Glackin, D. L., Linsky, J. L., Mango, S. A., \& Bohlin, J. D. 1978, ApJ, 222, 707

Gouttebroze, P., \& Labrosse, N. 2000, Sol. Phys., 196, 349

Gouttebroze, P., \& Labrosse, N. 2009, A\&A, 503, 663

Gunár, S., Heinzel, P., Schmieder, B., Schwartz, P., \& Anzer, U. 2007, A\&A, 472,929

Gunár, S., Heinzel, P., Anzer, U., \& Schmieder, B. 2008, A\&A, 490, 307

Harrison, R. A., Sawyer, E. C., Carter, M. K., et al. 1995, Sol. Phys., 162, 233

Heinzel, P., Schmieder, B., \& Tziotziou, K. 2001, ApJ, 561, L223

Heinzel, P., Schmieder, B., Fárník, F., et al. 2008, ApJ, 686, 1383

Kosugi, T., Matsuzaki, K., Sakao, T., et al. 2007, Sol. Phys., 243, 3

Labrosse, N., \& Gouttebroze, P. 2001, A\&A, 380, 323

Labrosse, N., \& Gouttebroze, P. 2004, ApJ, 617, 614

Labrosse, N., Vial, J.-C., \& Gouttebroze, P. 2008, Ann. Geophys., 26, 2961

Labrosse, N., Heinzel, P., Vial, J.-C., et al. 2010, Space Sci. Rev., 151, 243

Landi, E., \& Young, P. R. 2010, ApJ, 714, 636

Lang, J., Kent, B. J., Paustian, W., et al. 2006, Appl. Opt., 45, 8689

Léger, L., \& Paletou, F. 2009, A\&A, 498, 869

Linsky, J. L., Glackin, D. L., Chapman, R. D., Neupert, W. M., \& Thomas, R. J. 1976, ApJ, 203, 509

Mackay, D. H., Karpen, J. T., Ballester, J. L., Schmieder, B., \& Aulanier, G. 2010, Space Sci. Rev., 151, 333

Mariska, J. T., Doschek, G. A., \& Feldman, U. 1979, ApJ, 232, 929

Mazzotta, P., Mazzitelli, G., Colafrancesco, S., \& Vittorio, N. 1998, A\&AS, 133, 403

Orrall, F. Q., \& Schmahl, E. J. 1976, Sol. Phys., 50, 365

Schmieder, B., Lin, Y., Heinzel, P., \& Schwartz, P. 2004, Sol. Phys., 221, 297

Schmieder, B., Chandra, R., Berlicki, A., \& Mein, P. 2010, A\&A, 514, A68

Schwartz, P., Heinzel, P., Anzer, U., \& Schmieder, B. 2004, A\&A, 421, 323

Török, T., Aulanier, G., Schmieder, B., Reeves, K. K., \& Golub, L. 2009, ApJ, 704,485

Wiik, J. E., Schmieder, B., \& Noens, J. C. 1994, Sol. Phys., 149, 51

Young, P. R., Del Zanna, G., Mason, H. E., et al. 2007a, PASJ, 59, 857

Young, P. R., Del Zanna, G., Mason, H. E., et al. 2007b, PASJ, 59, 727 